\title{
High Expression of Genes for Calcification-regulating Proteins in Human Atherosclerotic Plaques
}

\author{
Catherine M. Shanahan, Nathaniel R. B. Cary, ${ }^{\star}$ James C. Metcalfe, ${ }^{\ddagger}$ and Peter L. Weissberg \\ Department of Medicine, Level 5, Addenbrooke's Hospital, Cambridge, CB2 2QQ United Kingdom; ${ }^{*}$ Department of Pathology, \\ Papworth Hospital, Papworth Everard, Cambridgeshire, United Kingdom; and ${ }^{\ddagger}$ Department of Biochemistry, Cambridge, \\ CB2 1QW United Kingdom
}

\begin{abstract}
Calcification is common in atheromatous plaques and may contribute to plaque rupture and subsequent thrombosis. However, little is known about the mechanisms which regulate the calcification process. Using in situ hybridization and immunohistochemistry we show that two bone-associated proteins, osteopontin (OP) and matrix Gla protein (MGP), are highly expressed in human atheromatous plaques. High levels of OP mRNA and protein were found in association with necrotic lipid cores and areas of calcification. The predominant cell type in these areas was the macrophage-derived foam cell, although some smooth muscle cells could also be identified. MGP was expressed uniformly by smooth muscle cells in the normal media and at high levels in parts of the atheromatous intima. Highest levels of this matrix-associated protein were found in lipid-rich areas of the plaque. The pattern of expression of these two genes contrasted markedly with that of calponin and SM22 $\alpha$, genes expressed predominantly by differentiated smooth muscle cells and whose expression was generally confined to the media of the vessel. The postulated function of $O P$ and MGP as regulators of calcification in bone and the high levels and colocalization of both in atheromatous plaques suggest they have an important role in plaque pathogenesis and stability. (J. Clin. Invest. 1994. 93:2393-2402.) Key words: osteopontin • matrix Gla protein • calponin - SM22 $\alpha$ • vascular smooth muscle
\end{abstract}

\section{Introduction}

Myocardial infarction occurs as a result of thrombosis on a fissured atherosclerotic plaque. Recent studies have shown that intimal tearing commonly occurs at the lateral margins of lipid pools or where there is significant calcification within the intima (1). Consistent with this, it has been shown that the presence of vascular calcification in asymptomatic men is associated with an increased risk of clinically significant coronary artery disease (2). Atheromatous plaques are composed predominantly of vascular smooth muscle cells (VSMCs), ${ }^{1}$ macro-

Address correspondence to Dr. C. Shanahan, Department of Medicine, Level 5, Addenbrooke's Hospital, Hills Road, Cambridge CB2 2QQ, United Kingdom.

Received for publication 9 June 1993 and in revised form 24 January 1994.

1. Abbreviations used in this paper: MGP, matrix Gla protein; OP, osteopontin; SM, smooth muscle; VSMC, vascular smooth muscle cell.

J. Clin. Invest.

(C) The American Society for Clinical Investigation, Inc.

$0021-9738 / 94 / 06 / 2393 / 10 \quad \$ 2.00$

Volume 93, June 1994, 2393-2402 phages and extracellular matrix but little is known of the processes that lead to calcification of the arterial wall or which cells are involved in its genesis.

Morphological studies have shown that VSMCs in atheromatous plaques differ from contractile VSMCs in the media in that they contain numerous synthetic organelles and few contractile filaments (3). These plaque VSMCs are known to synthesize matrix proteins which contribute to the composition of the plaque, therefore the proteins they produce may determine the nature of the resulting atherosclerotic lesion. To identify potential markers of VSMC phenotypes and to predict the role of VSMCs in healthy and diseased vessels, we used the technique of differential cDNA screening of a rat VSMC cDNA library to identify genes highly expressed in differentiated, contractile VSMCs but barely expressed in proliferating VSMCs and vice versa (4). Amongst the genes most highly expressed in contractile VSMCs were those encoding calponin, a smooth muscle-specific protein associated with the contractile filaments and SM22 $\alpha$, a smooth muscle-specific protein of unknown function $(5,6)$. From proliferating VSMCs two cDNAs were identified encoding the proteins osteopontin (OP) and matrix $\gamma$-carboxyglutamic acid (Gla) containing protein (MGP). OP mRNA was barely detectable in differentiated, nonproliferating VSMCs but was upregulated when the cells proliferated, while MGP mRNA was expressed at low levels in differentiated VSMCs but was substantially upregulated in proliferating VSMCs. Both these genes were originally isolated from developing bone and are thought to play a role in the regulation of calcification during bone development and remodeling $(7,8)$.

In this study we have investigated the expression of the genes for calponin, SM22 $\alpha$, OP, and MGP in human atherosclerotic lesions to determine whether these genes, which were identified from rat VSMCs in cell culture, are expressed in human vessels and whether their patterns of expression conform to those predicted by our in vitro study. Our findings confirm that calponin and SM22 $\alpha$ are expressed by differentiated VSMCs and that both OP and MGP are highly expressed in atherosclerotic lesions. However, the cells responsible for most of the expression of OP and MGP in the plaque were the macrophage-derived foam cells. Our findings demonstrate a close association between the expression of OP and MGP with calcification within lipid-rich areas of the plaque and allow us to propose a mechanism for plaque calcification.

\section{Methods}

In situ hybridization. Fresh coronary arteries $(n=20)$ were obtained from nine recipient hearts excised at the time of orthotopic transplantation. Samples were mounted in Tissue-Tek OCT embedding compound (Miles Ames Division, Inc., Elkart, IN), snap frozen in liquid nitrogen and subsequently stored at $-70^{\circ} \mathrm{C}$ before sectioning. Sections 
were cut (8-10 $\mu \mathrm{m})$ and mounted onto gelatinized slides, refrozen and stored at $-70^{\circ} \mathrm{C}$ until use. The sections were processed for in situ hybridization according to Boehm et al. (9) except hybridization was carried out using ${ }^{35} \mathrm{~S}$-labeled sense and anti-sense riboprobes generated by in vitro transcription from the T3 and T7 promoters of Bluescript SK-linearized plasmids (Stratagene, La Jolla, CA). The human osteopontin, (OPN) and human matrix Gla, (GLA) probes were transcribed from full-length cDNA clones of 1.4 and $0.7 \mathrm{~kb}$, respectively, and were obtained from the ATCC/NIH repository (Rockville, MD). The probe for rat calponin (4A9) was a $1.4 \mathrm{~kb}$ full length cDNA and for rat SM22 $\alpha$ (3RF10) a 1.0-kb cDNA clone as previously reported (4), and shown by Northern analysis to cross-hybridize with human mRNA under the conditions described. After 3 wk of exposure slides were developed, stained with haemotoxylin-eosin, and mounted.

Immunohistochemistry. Immunohistochemistry was performed on sections adjacent to those used for in situ hybridization using avidinbiotin immunoperoxidase enhancement as described by Hsu et al. (10). VSMCs were identified with a mouse monoclonal antibody to human $\alpha$-smooth muscle (SM)-actin (Dako, M815, dilution 1:25), (Dako Corp., Carpinteria, CA). Macrophages were identified with a mouse monoclonal antibody to CD68 (Dako, macrophage: EMB11, dilution 1:20), (Dako Corp.) which stains all tissue macrophages. $T$ cells were identified with an antibody to CD3 (Unipath PanT, dilution 1:50), (Unipath, Nepean, Ontario, Canada) and endothelial cells were identified with antibody QBend 10, (Unipath, QBend10, dilution 1:50), (Unipath), as described by Rovani et al. (11). The MGP antibody was a gift from Dr. R. F. Loeser, The Bowman Gray School of Medicine, Wake University (Raleigh, NC). This polyclonal antibody was raised in rabbits immunized with a 19-amino acid synthetic peptide from the bovine MGP sequence as described (12) and diluted 1:100. The OP antibody $\left(M P I I B 10_{1}\right)$ was a mouse monoclonal antibody raised against rat bone OP obtained from the National Institute of Child Development Hybridoma Bank 9 (Iowa City, IA) and diluted 1:200. Controls were performed substituting the primary antibody with either PBS or an irrelevant antibody.

Lipid and calcium were demonstrated using Oil Red ' $O$ ' and von Kossa stain, respectively.

\section{Results}

18 atherosclerotic lesions from various excised coronary arteries were studied. Serial sections from these lesions and from two normal coronary arteries were used for in situ hybridiza-

Table I. Expression of OP and MGP MRNA in Atherosclerotic Plaques

\begin{tabular}{|c|c|c|c|c|c|c|c|c|c|}
\hline & & & & & & \multicolumn{4}{|c|}{ Gene expression } \\
\hline \multicolumn{6}{|c|}{ Lesion description } & \multicolumn{2}{|c|}{ OP } & \multicolumn{2}{|c|}{ MGP } \\
\hline Vessel & Lesion & Type & Media $^{*}$ & Lipid $^{*}$ & Calcium ${ }^{8}$ & Media & Intimall & Media' & Intima \\
\hline A: Mid RC & 1 & Fatty streak & $\mathbf{N}$ & - & - & - & - & + & $+\mathrm{SM}$ \\
\hline Prox RC & 2 & Fatty streak & $\mathbf{N}$ & - & - & - & - & + & $+\mathbf{S M}$ \\
\hline B: Prox LAD & 3 & Fatty streak & $\mathbf{N}$ & $3^{* *}$ & + & - & $+++\mathbf{M}$ & ++ & $+++\mathrm{M}, \mathrm{SM}$ \\
\hline Mid LAD & 4 & Fatty streak & $\mathbf{N}$ & $5^{* *}$ & + & +++ foci-M & $+++\mathbf{M}$ & ++ Foci-M, SM & $+++\mathrm{M}, \mathbf{S M}$ \\
\hline Prox RC & 5 & Normal & $\mathbf{N}$ & - & - & - & - & + & $+\mathrm{SM}$ \\
\hline C: Prox RC & 6 & Normal & $\mathrm{N}$ & - & - & - & - & ++ & $++\mathbf{S M}$ \\
\hline OMI & 7 & Fibro/lipid & $\mathrm{T}$ & 1 & + & - & $++M$ & + & $+\mathbf{M}, \mathbf{S M}$ \\
\hline $\mathrm{CX}$ & 8 & Fibro/lipid & $\mathbf{T}$ & + & + & - & $+\mathbf{M}$ & ++ & $+++\mathbf{M}, \mathbf{S M}$ \\
\hline D: Prox LAD & 9 & Fibro/lipid & $\mathbf{T}$ & 2 & + & - & $++M$ & ++ & $+++\mathrm{M}, \mathrm{SM}$ \\
\hline Mid RC & 10 & Fibrous & $T$ & 2 & + & - & $-\ddagger$ & + & $+\mathrm{M}, \mathbf{S M}$ \\
\hline E: Prox RC & 11 & Fibro/lipid & $\mathbf{N}$ & 1 & + & +++ Foci-M & $+++\mathbf{M}$ & ++ Foci-M, SM & $+++\mathbf{M}, \mathbf{S M}$ \\
\hline F: Prox LAD & 12 & Fibro/lipid & $\mathbf{T}$ & 2 & + & - & $+++\mathbf{M}$ & + & $+++\mathrm{M}, \mathrm{SM}$ \\
\hline Dist LAD & 13 & Fibro/lipid & $\mathbf{N}$ & 1 & + & - & $+++\mathbf{M}$ & + & $+M$ \\
\hline G: Prox RC & 14 & Fibrous & $\mathrm{T}$ & + & - & - & $-\ddagger$ & + & ++ SM-foci \\
\hline Prox LAD & 15 & Fibro/lipid & $\mathbf{T}$ & 1 & +++ & - & $+++\mathbf{M}$ & + & $+++\mathrm{M}, \mathrm{SM}$ \\
\hline OMI & 16 & Fibro/lipid & $\mathbf{T}$ & 2 & +++ & - & $+++\mathbf{M}$ & + & $++\mathrm{M}, \mathrm{SM}$ \\
\hline H: Mid RC & 17 & Fibro/lipid & $\mathbf{T}$ & 2 & +++ & - & $+++\mathrm{M}$ & + & $++\mathbf{M}, \mathbf{S M}$ \\
\hline L main & 18 & Fibro/lipid & $\mathbf{T}$ & 4 & +++ & - & $+++\mathrm{M}$ & + & $+++\mathbf{M}, \mathbf{S M}$ \\
\hline Prox LAD & 19 & Fibrous & $\mathbf{T}$ & 1 & +++ & - & $-\ddagger$ & + & $+\mathrm{M}, \mathrm{SM}, \mathrm{E}$ \\
\hline I: $\quad$ Mid RC & 20 & Fibro/lipid & $\mathbf{T}$ & 4 & +++ & - & $+++\mathbf{M}$ & + & +++ SM, M, E \\
\hline
\end{tabular}

$A-I$ refer to patients from whom the heart was removed and vessels excised: $R C$, right coronary artery; $L A D$, left anterior descending coronary artery; $O M I$, first obtuse marginal branch of the circumflex coronary artery; $C X$, circumflex coronary artery; $L$ main, left main coronary artery; Dist, distal; Prox, proximal. * The media of each vessel is described as thinned $(T)$ if the width of the artery media involved in the plaque was less than $50 \%$ of the normal uninvolved media $(N)$. ' ${ }^{\prime}$ Lipid was detected in the lesions using Oil Red ' $O$ ' staining. Numbers refer to the number of discreet lipid pools in the plaque. The + symbol denotes positive staining for lipid in the absence of discreet lipid pools. Lipid in these lesions was in foam cells. ** These lipid pools were very small areas of lipid accumulation within an early intimal thickening. ${ }^{\star} \mathrm{Calcium}$ was detected in the lesions using von Kossa staining. Calcified regions varied from heavily calcified necrotic cores $(+++)$ to small granular calcium deposits detectable within the matrix or associated with lipid $(+)$. "OP expression was highest in foam-filled macrophages which were the predominant cell type at the edge of lipid-pools. In medial foci of OP expression macrophages were expressing the OP mRNA however expression by adjacent VSMCs could not be excluded. $M$, macrophages. $(+++)$ High levels of expression in lesion, $(++)$ mid levels of the expression in lesion, $(+)$ low levels of expression in lesion, $(-)$ no expression detectable. ${ }^{\ddagger}$ These intimal lesions were hypocellular and composed mainly of extracellular matrix. 'MGP was expressed in the media of vessels predominantly by VSMCs and in the intima by macrophages ( $M$ ), smooth muscle cells $(S M)$, and endothelial cells $(E)$. Cell types were identified by specific immunohistochemical staining on serial sections of the lesions as described in the text. 
tion and immunohistochemistry. Lesions ranged from early fatty streaks characterized by focal, eccentric intimal thickening, and some macrophage infiltration, to later fully developed fibro/lipid plaques with lipid pools and associated necrotic and calcified areas. Three lesions from cases with advanced atherosclerosis were largely devoid of intimal cells and were classified as fibrous. It was noted that in a number of vessels the media adjacent to the atheromatous plaque was thinned. Table I summarizes the composition of the vascular lesions studied (1-20 including two normal coronary arteries) and shows the gene expression patterns for OP and MGP in these lesions.

Calponin and $S M 22 \alpha$. Results from our previous study predicted that calponin and SM22 $\alpha$ would be expressed predominantly in medial VSMCs. Consistent with this prediction we found that expression of calponin was always confined to the media of normal and diseased vessels (Fig. 1). There was no detectable expression in the intima, even in areas where positive staining for a-SM actin identified many of these intimal cells as VSMCs. SM22 $\alpha$ was highly expressed in the media and was also expressed in some but not all of the a-SM actin-positive VSMCs of the fibrous cap and other areas of the intima (Fig. 1). Both genes were also clearly expressed in the medial VSMCs of small adventitial vessels (Fig. 2). The level of expression of calponin and SM22 $\alpha$ in the media appeared to be related to the degree of medial thinning. Generally, expression of calponin and SM22 $\alpha$ was high in morphologically normal medias, even when associated with a lesion, whereas there was little or no detectable calponin mRNA and low levels of SM22 $\alpha$ mRNA in thin or damaged medias. In some thinned medias, where calponin mRNA was absent, SM22 $\alpha$ expression
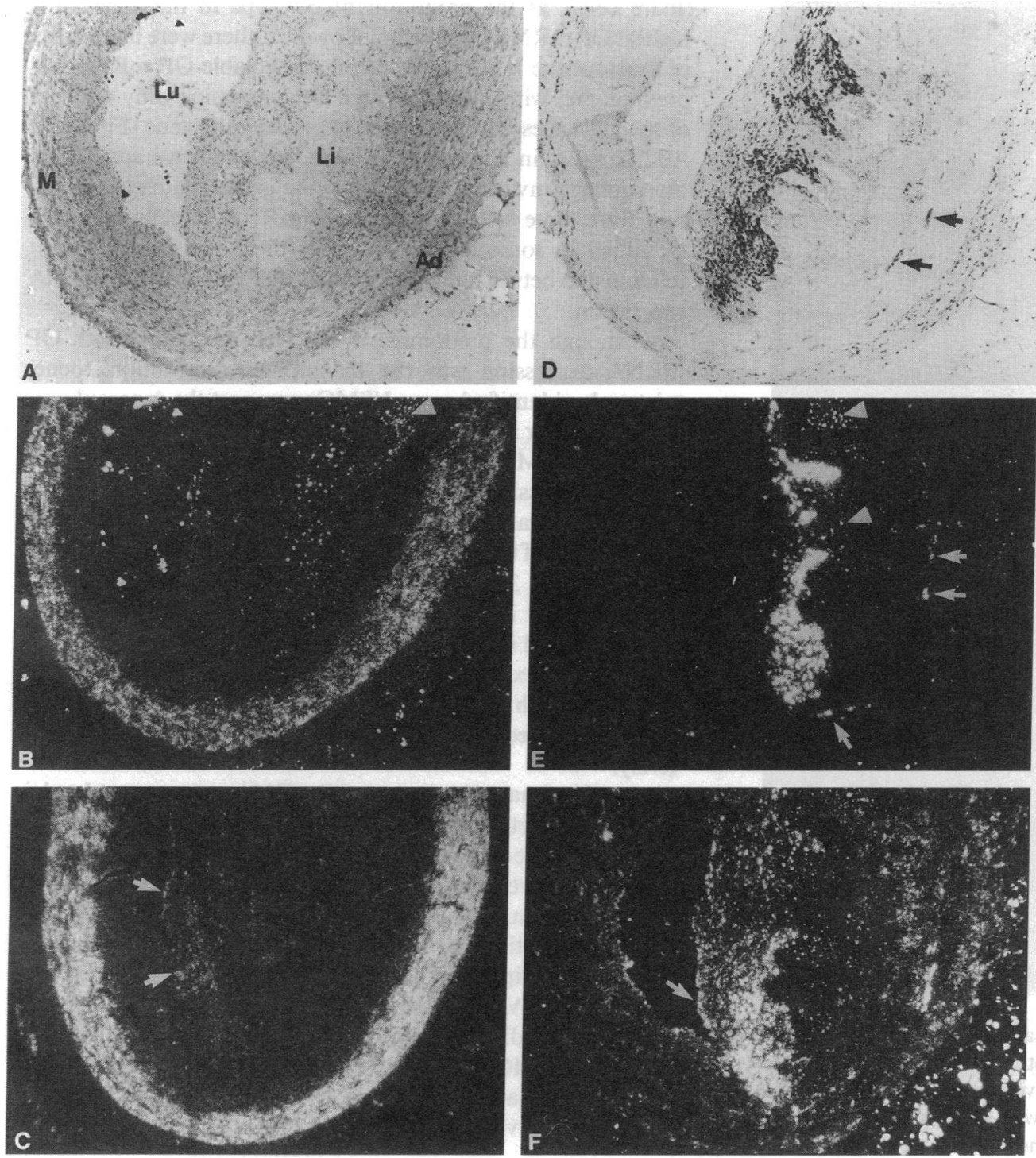

Figure 1. Serial sections of an advanced atheromatous plaque (lesion 11, Table I) from a right coronary artery. $(A)$ Haemotoxylin-eosin stain of the lesion showing a large necrotic lipid core $(L i)$, significantly narrowed lumen $(L u)$, and a normal media $(M)$ and adventitia $(A d)$. Von Kossa staining identified areas of calcification in this lesion in association with the lipid pool. Some of these areas are marked by arrowheads in $B$ and $E$ where the calcium is seen as white fluorescence under dark-field illumination. (B) Dark-field photomicrograph showing in situ hybridization for calponin. The expression of calponin is confined to the media of the vessel. (C) Dark-field photomicrograph showing in situ hybridization for SM22 $\alpha$. Expression is highest in the media but some expression is detectable in smooth muscle cells of the fibrous cap (arrows). (D) Immunohistochemical staining for macrophages. This shows that the major areas of macrophage infiltration are in the shoulder region and around the periphery of the necrotic lipid core. There are also small isolated areas of macrophage infiltration in the media (arrows) and numerous adventitial macrophages. $(E)$ Dark-field photomicrograph showing in situ hybridization for OP. Expression is highest in the shoulder region and around the periphery of the necrotic lipid core. Isolated ex-

pression is also present in areas of the media (arrows) which were also sites of macrophage infiltration (see corresponding regions in D). Note the absence of OP expression in the areas of the plaque composed of predominantly VSMCs (i.e., the media and fibrous cap). $(F)$ Dark-field photomicrograph showing in situ hybridization for MGP. Hybridization for MGP was detected in the adventitia, media, and intima. Expression is highest in the shoulder region of the lesion in association with macrophages and smooth muscle cells. There is also high expression of MGP in the cells lining the lumen of the vessel (arrows) and in the same medial macrophages that express high levels of OP mRNA. No hybridization was detectable in controls (sense probes corresponding to all anti-sense probes used) in a further set of serial sections (not shown). 

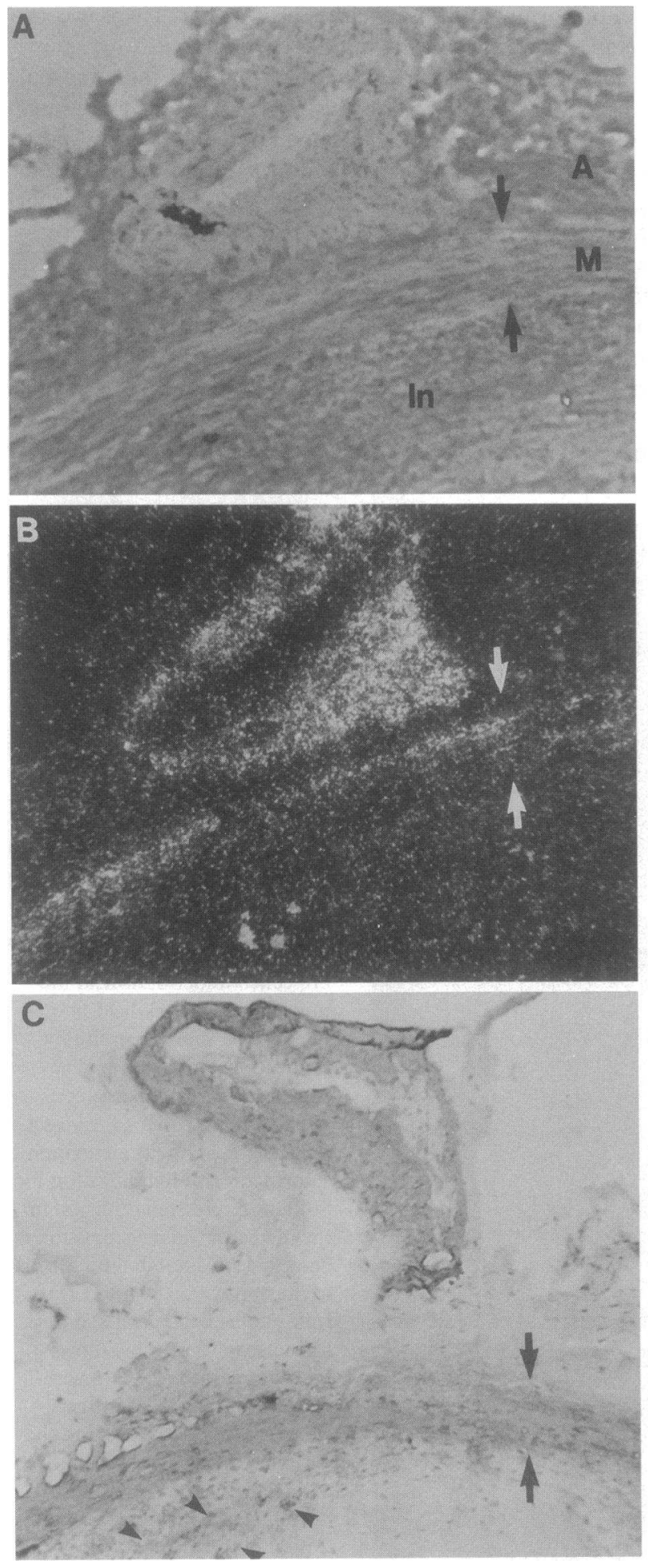

Figure 2. Atheromatous lesion from a right coronary artery including a small adventitial vessel. $(A)$ Haemotoxylin-eosin stain demonstrating that the media $(M)$ of the large vessel adjacent to the intimal lesion $(I)$ is thinned (defined by arrowheads) compared with media uninvolved in lesion (not shown). The adjacent adventitial vessel $(A)$ has a normal media. $(B)$ Dark-field photomicrograph of the same section showing in situ hybridization for SM22 $\alpha$. Note the expression of SM22 $\alpha$ throughout the media of the adventitial vessel but patchy expression in the media of the larger vessel with only some of the medial smooth muscle cells expressing SM22 $\alpha$ mRNA. (C) a-SM actin staining of a section (the adventitial vessel is slightly displaced) adjacent to the section shown in $A$ demonstrating that not all a-SM was patchy with some of the apparantly normal a-SM actin-positive VSMCs expressing no detectable SM22 $\alpha$ mRNA adjacent to VSMCs expressing high levels of SM22 $\alpha$ mRNA (Fig. 2). This heterogeneous pattern of SM $22 \alpha$ expression was also found in the intima with only some cells in areas strongly positive for a-SM actin protein expressing SM22 $\alpha$ mRNA.

Osteopontin $m R N A$. No OP mRNA was detectable in normal vessels (Table I). High OP mRNA expression was found in 13 of the 18 atherosclerotic lesions examined with most expression in advanced fibro/lipid plaques at the margins of lipid pools and in the shoulder region of the fibrous cap (Fig. 1). OP mRNA was also expressed in two of the four early lesions studied (lesions 3 and 4, Table I). Those early lesions which expressed OP differed from the early lesions which did not in that they contained a number of very small lipid pools with which the OP expression was associated. Immunohistochemistry identified macrophages, particularly lipid-laden macrophages (foam cells), as the predominant cell type in the areas with highest OP mRNA expression. However, there were large areas of macrophage infiltration with no detectable OP mRNA expression and within areas of high OP expression only a subset of macrophages appeared to be expressing the gene (Fig. $3 \mathrm{~B}$ ). Additionally, in some vessels there was extensive adventitial macrophage invasion but in only one isolated area of one lesion were these cells expressing OP mRNA. T cells were also identified in some lesions, however there was no consistent relationship between the distribution of $T$ cells and OP mRNA expression.

Although the predominant cell type associated with OP mRNA expression was the macrophage, immunohistochemistry also identified some VSMCs amongst the macrophages in the shoulder regions of the plaques. It was therefore possible that some VSMCs within these areas were also contributing to the OP expression (Fig. 4). In two lesions (lesions 4 and 11, Table I), several foci of OP $m$ RNA expression were identified in the media of the vessel where VSMCs were the predominant cell type (Fig. $3 \mathrm{~A}$ ). However, infiltrating macrophages were always present in these areas. Thus, OP mRNA expression in the intima and media always coincided with macrophage infiltration. Also, expression of OP mRNA in the media was always associated with isolated areas of lipid and/or micro-calcification as identified by Oil Red 'O' and von Kossa staining, respectively.

In all intimal lesions where OP mRNA was expressed, calcification was detectable by von Kossa staining, usually adjacent to or within necrotic lipid cores (Table I). OP mRNA was not detected in seven vessels; four of which contained no lipid pool or calcification. The other three lesions contained lipid with some calcification but few detectable intimal cells in association with the lipid and calcium in the sections examined.

MGP $m R N A$. Unlike OP, MGP mRNA was clearly expressed by medial and intimal VSMCs (Fig $3 E$ ). In atherosclerotic lesions MGP mRNA was expressed by most cells including intimal macrophages, endothelial and VSMCs lining the lumen of the vessel and adventitial macrophages (Fig. 1). In

actin positive cells express SM22 $\alpha$ mRNA. Some intimal smooth muscle cells (arrows) are clearly not expressing SM22 $\alpha$ mRNA. Calponin was expressed throughout the media of the adventitial vessel but was not detectable in the thinned media of the large vessel (not shown). 

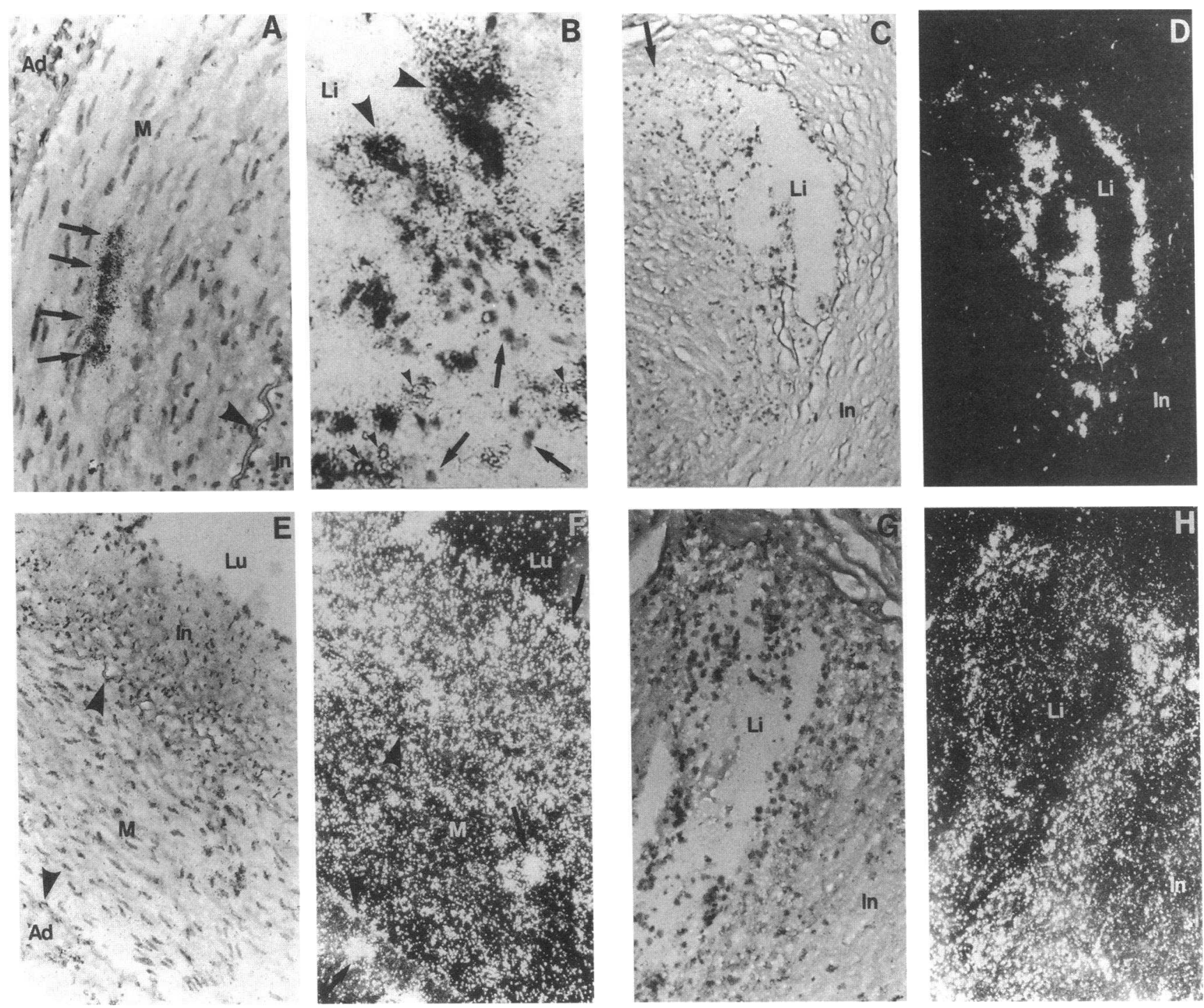

Figure 3. Photomicrographs showing in situ hybridization for OP $(A-D)$ and MGP $(E-H)$ in human atheromatous plaques. $(A)$ A focus of OP expression in the media (arrows). OP is expressed by infiltrating macrophages and possibly the associated VSMCs. Cell types were identified by immunohistochemistry to adjacent sections (not shown). Oil Red ' $O$ ' and von Kossa staining showed that both lipid and microcalcification where present in this area. These same cells were also expressing high levels of MGP mRNA. Arrowhead indicates internal elastic lamina. $A d$, adventitia; $M$, media; In, intima. $(B)$ OP expression in macrophages at the edge of a lipid pool. Large arrowheads show areas of high OP expression while arrows indicate a subset of macrophages surrounding a lipid pool $(L i)$ that are not expressing OP mRNA. Small arrowheads indicate calcium. $(C$ and $D)$ Light and dark field illumination of OP expression in macrophages at the periphery of a lipid pool in a fibrous plaque. Arrow indicates an area containing macrophages that are not expressing OP mRNA. $(E$ and $F)$ Light and dark-field illumination of MGP expression in a plaque showing some expression in all cells and high expression in adventitial macrophages (arrows), medial smooth muscle cells and some associated medial macrophages (arrows) and, the endothelial (identified by immunohistochemistry) and smooth muscle cells lining the edge of the lumen (arrows). Arrowheads delineate the media of the vessel. ( $G$ and $H$ ) MGP expression in the same lesion and lipid pool as shown in $C$ and $D$. Note that MGP expression is in all cells (macrophages and smooth muscle cells) of the plaque including those lining the periphery of the lipid pool, but levels of expression per cell are generally lower than those for OP.

vessels with medial thinning MGP mRNA was uniformly expressed in the remaining medial cells. The highest levels of MGP mRNA were expressed by both VSMCs and macrophages in lipid-rich areas of the plaques. Although MGP and OP mRNAs were both expressed in lipid-rich areas of the plaque, their expression was not completely coincidental. MGP mRNA was generally less highly expressed than OP mRNA at the periphery of the lipid pools (Fig. 3); the MGP mRNA being most highly expressed by cells not directly adjacent to, but further back from the periphery of the lipid pools, see Fig. 1, $E$ and $F$. In the two lesions which had medial foci of
OP mRNA expression (lesions 4 and 11, Table I), MGP mRNA was also expressed at a high level in the same medial cells. In the fibrous lesions where OP expression was undetectable MGP mRNA was detectable in most of the few remaining intimal cells.

$O P$ and MGP immunohistochemistry. Having detected mRNA for both OP and MGP in the atheromatous plaques we went on to look for the respective proteins using immunohistochemistry. Both OP and MGP proteins were detectable and colocalized in the lipid-rich areas of the plaque (Fig. 5). Staining for OP protein was highest in macrophages associated with 

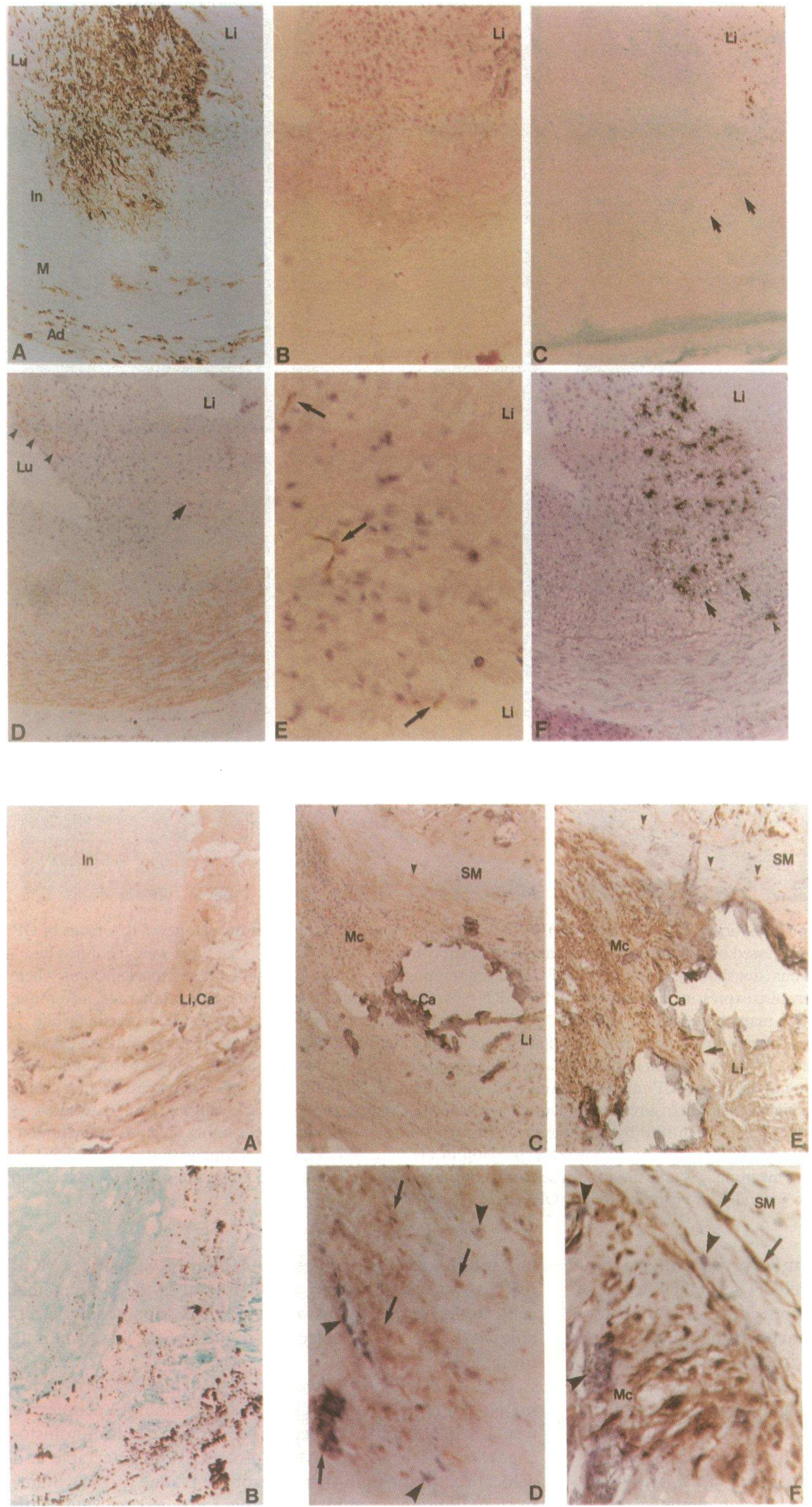
lipid pools corresponding to areas of highest expression of the gene. The protein was mostly cell-associated with some matrix staining in areas of greatest macrophage infiltration, particularly at the edge of lipid pools. A larger number of macrophages were associated with OP protein than were expressing the mRNA, suggesting that the mRNA expression may be episodic, possibly in response to a particular stimulus, with the resulting protein remaining in or around the cell after the message is downregulated. As shown in Fig. 5, the highest levels of OP protein were found in association with macrophage-derived foam cells on the edge of lipid pools adjacent to calcification. In all the lesions examined macrophages which had penetrated the lipid core of the plaques were always positive for $\mathrm{OP}$ protein.

There was a low level of matrix-associated OP protein staining in the normal media in areas where the only cells present were VSMCs. However, as shown in Fig. 1, these medial VSMCs did not appear to express OP mRNA. Also, a number of VSMCs in the intima of the plaque stained positively for OP protein (Fig. $5 F$ ) where again no mRNA was detected. While some of this staining was matrix-associated, particularly in fibrous areas of the plaque, most was cell-associated. Thus there appears to be a much wider distribution of the OP protein than would have been predicted by the in situ hybridization data presented above.

In contrast to OP protein which was often cell-associated, MGP protein was mostly matrix associated. The protein could be detected at low levels in the normal media and adventitia but staining was highest in the intima where gene expression was also highest. Like OP, the greatest staining for MGP protein occurred in association with lipid-rich, calcified areas of the plaque (Fig. 5). In these areas the protein appeared in dense clumps in the matrix especially in association with cell debris, implying release of the protein from dead or dying cells. There was a low level of matrix and cell-associated staining for MGP protein detectable at the lumenal edge of most lesions. This pattern of distribution of the protein in the plaques was very similar to that predicted from the pattern of gene expression. However, there was some evidence to suggest that the protein was concentrated in the plaque matrix in lipid-rich areas. An example of this is shown in Fig. $5 C$ where there are intimal VSMCs next to an area rich in oil red ' 0 '-positive macrophages. The level of MGP mRNA expression is uniform over this region whilst the protein is concentrated in areas containing lipid and cellular debris.

\section{Discussion}

Expression of markers of differentiated VSMCs. We have shown that the genes for calponin and SM22 $\alpha$ were both highly expressed in the media of healthy human vessels but were downregulated in intimal VSMCs in atheromatous lesions, findings which are consistent with our previous in vitro study (4). The level of expression of calponin and SM22 $\alpha$ in medial cells varied between lesions and was reduced when the media was thinned. Medial thinning is a feature of atheromatous disease which is poorly understood but which may contribute to weakening of the vessel wall. It is therefore possible that some apparently normal VSMCs (as judged by their appearance and a-SM actin staining) in the thinned media may be partially de-differentiated and have impaired contractile function.

It appears that the expression of genes for calponin, SM22 $\alpha$ and possibly a-SM actin are not coordinately regulated since there were many a-SM actin-positive cells, both in thinned media and the intima, which did not express either calponin or SM22 $\alpha$ mRNA. Furthermore, calponin mRNA was often downregulated in medial cells which still expressed SM22 $\alpha$ mRNA and a-SM actin protein suggesting that calponin is a sensitive marker of the differentiated phenotype and that loss of expression of this gene is an early event in vascular disease.

Expression of $O P$ in atheromatous plaques. Our study has demonstrated the presence of OP mRNA and protein in ather-

Figure 4. Details of the cell types and regions of the plaque that express OP mRNA. $(A-F)$ Serial sections showing high power views $(\times 200)$ of the shoulder region of the same plaque as shown in Fig. 1. (A) Immunohistochemistry with anti-CD-68 antibody showing the location of macrophages within the plaque. Macrophage infiltration can be seen in the adventitia $(A d)$ and media $(M)$ but is greatest in the shoulder region of the intimal $(I n)$ lipid pool $(L i)$. (B) Oil Red 'O' staining showing that the areas of greatest macrophage infiltration in the shoulder region of the plaque contain extensive lipid deposits. $(C$ ) von Kossa staining showing granular calcification associated with the lipid-rich necrotic core. Arrows indicate areas of granular and micro-calcification extending into the shoulder region. $(D)$ Immunohistochemistry for a-SM actin showing the VSMCs of the plaque. Most VSMCs are confined to the media or are just on the intimal side of the internal elastic lamina, however, there are some VSMCs in the shoulder region of the plaque (arrow) and fibrous cap (arrowheads). $(E)$ Higher power view $(\times 800)$ of the shoulder region of the plaque arrowed in $D$ showing the presence of isolated VSMCs (arrows) amongst infiltrating macrophages. $(F)$ Light-field illumination showing OP expression in the shoulder region and periphery of the lipid pool. Arrows indicate OP expression in the same region as the microcalcification in $C$. Arrowhead indicates an area of OP expression near the internal elastic lamina in which the predominat cell type is VSMCs although macrophage infiltration is also present at this site (see $A$ ).

Figure 5. Immunohistochemistry showing OP and MGP protein in human atheromatous plaques. $(A)$ Immunohistochemistry for MGP in a region of a heavily calcified lesion shown by von Kossa staining $(B)(\times 200)$. Note that the greatest accumulation of protein is in the matrix around the calcified $(\mathrm{Ca})$ areas which were also lipid-rich $(\mathrm{Ll})$. The cellular region of the intima $(\mathrm{In})$ which is composed mainly of VSMCs contains less protein even though mRNA expression for MGP in this area was equal to that in the calcified regions. ( $C$ and $E$ ) MGP and OP protein in the same lesion $(\times 200)$. (C) Diffuse staining for MGP is found in most areas of the lesion except the band of smooth muscle cells $(S M)$ above the large calcified, necrotic core. There are regions of localized MGP staining in areas surrounding the smooth muscle (arrowheads). (E); Staining for OP protein is associated with most of the macrophages in the lesion $(M c)$ and with macrophages penetrating into the calcified, lipid core (arrows). The band of smooth muscle cells $(S M)$ contains a few positive cells (arrowheads). $(D$ and $F)$ High power views $(\times 400)$ of MGP $(D)$ and OP $(F)$ protein within the calcified areas of the lesion (arrowheads indicate areas of calcium). (D) MGP is matrix associated with localized areas of heavy staining (arrows) in regions with calcium and debris. $(F)$ OP is associated with both macrophages and smooth muscle cells with the protein clearly defining the shape of the different cell types. In the area shown in $F$ there was high expression of OP mRNA in the macrophages that stain positive for OP however, no detectable OP mRNA expression was seen in the OP protein positive VSMCs (arrows). Negative controls did not stain for either antibody (not shown). 
omatous plaques where expression of the gene was more closely associated with macrophages than VSMCs at sites of calcificaton. These findings are consistent with those of Giachelli et al. (13) who found high levels of OP protein in calcified human atherosclerotic plaques, particularly in association with macrophages, but also associated with some VSMCs in the the same vicinity. However they did not perform in situ hybridization studies on their lesions and were therefore unable to demonstrate the source of OP. Our in situ hybridization results clearly show that macrophages express high levels of $O P$ mRNA but we were unable conclusively to demonstrate $O P$ mRNA expression in VSMCs. This was somewhat surprising since previous studies by ourselves and others have shown that proliferating rat VSMCs express high levels of OP mRNA in vitro $(4,14)$. Also, Giachelli et al. (13) showed that proliferating VSMCs in the rat carotid artery after balloon-induced intimal injury express high levels of OP mRNA and protein and we have observed a similar increase in OP mRNA expression by proliferating VSMCs in the same model (our own unpublished observations). Therefore, there can be no doubt that OP mRNA is upregulated in proliferating rat VSMCs in vitro and in vivo.

As yet, we have no clear evidence for OP mRNA expression by VSMCs in human vascular lesions and our rat and human data would seem to be at variance. However, there are a number of possible explanations for the apparent lack of OP mRNA expression by human VSMCs. We and others have shown, by Northern analysis, that OP mRNA is present at low levels in the normal rat aorta and carotid arteries $(4,13,14)$. Consistent with this Giachelli et al. demonstrated low levels of OP protein in the media of the rat carotid artery, yet in situ hybridization studies on the same tissues failed to demonstrate medial OP mRNA expression. We have also demonstrated low levels of expression of OP mRNA in normal human media by Northern analysis (our own unpublished observations) and failed to detect OP mRNA in the same tissue by in situ hybridization. Therefore, it seems likely that human medial VSMCs express OP mRNA at too low a level to be detected by in situ hybridization but at high enough levels to allow the protein to accumulate in the media. In the rat model of intimal injury there is unequivocal VSMC proliferation and sufficient upregulation of OP mRNA to be readily detected by in situ hybridization (13). However, in mature human atherosclerotic plaques the number of VSMCs that are proliferating is known to be very low (15). Therefore, if proliferation of human VSMCs is associated with upregulation of OP mRNA, it is not particularly surprising that little OP mRNA expression was seen in intimal VSMCs. Studies of restenotic lesions following therapeutic angioplasty, where VSMC proliferation is thought to be the main pathological event, might reveal OP mRNA expression by VSMCs. A recent communication by O'Brien et al. (16) suggests that this may be so (16).

Our results suggest that macrophages can retain OP protein after mRNA expression has ceased; only some macrophages in areas highly positive for OP protein were found to be expressing the mRNA. This raises the issue of the temporal relationship between expression of the mRNA and the protein. It is possible that VSMCs expressed high levels of OP mRNA at an earlier stage in the evolution of the plaques and that we missed this 'window' in our studies. Assuming a long half-life for the protein in the plaque, this would explain the VSMC-associated OP protein staining in the absence of high levels of mRNA.
Finally, it is possible that, unlike rat cells, proliferating human VSMCs do not express high levels of OP mRNA. We have preliminary evidence that cultured human VSMCs do not appear to upregulate OP mRNA (our own unpublished observations). The OP protein associated with intimal VSMCs in the lesions may be derived from another source. Giachelli et al. (13) suggest that the OP protein in the plaques may be derived from the circulation as this protein is present in blood plasma. However, our in situ hybridization results show that macrophages are a major source of OP in the plaque. Therefore, the OP protein associated with VSMCs may have been synthesised by macrophages and become bound to VSMCs. The cell adhesion RGDS (Arg-Gly-Asp-Ser)-domain present in OP would enable it to bind to any cell type expressing a receptor for this sequence (8).

Expression of MGP in atheromatous plaques. Our results show that high levels of mRNA and protein for MGP are expressed in human atherosclerotic plaques by both macrophages and VSMCs. Although MGP mRNA was present in the normal media, its expression was greatest in the atheromatous intima. The function of MGP in the healthy vessel wall is unknown. However, it is expressed in other soft tissues where its function may be to clear extracellular calcium and protect against tissue calcification $(12,17)$. In the atheromatous plaque, however, MGP may become trapped in the extracellular matrix, particularly bound to lipid where its affinity for calcium may predispose to tissue calcification. The protein staining we observed was similar to that observed in the newborn monkey articular-cartilage complex where all regions that ultimately undergo endochondral ossification stain deeply for MGP in the matrix (12). Additionally, studies in cynamolgous monkey coronary artery atherosclerotic plaques have demonstrated the presence of matrix-associated MGP within the plaque (R. F. Loeser, personal communication).

This is the first report of MGP expression by macrophagederived foam cells, but since not all macrophages within the lesion expressed the mRNA, the stimulus for its expression is unknown. It is possible that uptake of lipid by these cells induces MGP expression. However, preliminary experiments showed that human macrophages in culture do not express MGP mRNA even after incubation with oxidized low density lipoprotein for up to $24 \mathrm{~h}$ (our own unpublished observations), suggesting that additional factors are required to induce its expression. Like OP, its function in macrophages may be to promote cell adhesion $(18,19)$. The cell adhesion properties of MGP appear to be mediated by its calcium-binding Gla residues. However it has recently been shown that, despite the absence of an RGDS-domain, its adhesion properties in vitro are inhibited by synthetic RGDS-containing peptides raising the possibility of an interation between OP (which contains an RGDS sequence) and MGP in mediating cell adhesion.

Role of OP and MGP in plaque calcification. OP is a calcium-binding protein originally isolated from bone, where one of its functions is to attract and bind osteoclasts to hydroxyapatite and collagen bridges (8). In bone and other tissues OP expression is clearly associated with calcification; e.g., it is the major protein component of urinary stones $(20,21)$. However, OP is also expressed in transformed cells and in a variety of tissues which do not normally calcify, where it may play a role in cell adhesion $(22,23)$. In macrophages it is thought to promote interactions with fibronectin via its RGDS domain thereby facilitating chemotaxis (19). OP is encoded by a single 
gene, although alternative exon usage and post-translational modifications both produce a variety of proteins with potentially different roles $(20,21)$. Additionally, studies in vitro have shown that OP gene products can exert either restrictive or permissive effects on the formation of calcium crystals, depending on environmental conditions (20).

MGP is a vitamin $\mathrm{K}$-dependent protein with a high affinity for hydroxyapatite which it can bind via $\gamma$-carboxylated Gla residues. It is highly expressed in developing bone and cartilage where it is thought to act as a regulator of mineralization (7, 17). It is expressed at a lower level in soft tissues including kidney, heart, and aorta $(4,17)$ where its role is not clearly understood. It has been shown that in warfarin-treated rats and human fetuses whose mothers have taken warfarin during pregnancy there is excessive mineralizion of cartilage plates. This is thought to be due to loss of $\gamma$-carboxylation of Gla residues in MGP as a result of vitamin $\mathrm{K}$ antagonism $(7,17)$, further supporting the idea that MGP is a regulator of mineralization. In cartilage, MGP expression is highest during the calcification period with much of the protein sequestered in matrix vesicles, membrane bound bodies composed of cellular debris or lipid, which are thought to be actively involved in the mineralization process $(12,24,25)$. Like OP, MGP exhibits variation in transcript size and undergoes posttranslational modifications suggesting that it may encode multiple protein products $(4,25,26)$. Gla-containing proteins have also been shown to be present in urinary stones (27). Importantly, it has been shown that the amount of Gla-containing proteins in atheromatous lesions correlates positively with the extent of calcification (28). An additional Gla-containing protein, named atherocalcin, has been isolated from atheromatous lesions but the origin of this little characterized protein is unknown (28).

It is probable that neither OP nor MGP alone can induce calcification as both are expressed in soft tissues that do not normally calcify and under experimental conditions OP cannot nucleate hydroxyapatite (29). However, it is possible the high expression of OP by infiltrating macrophages and the presence of Gla-containing proteins together provide an environment which favors calcium crystal formation. Release of these calcium-regulating proteins by senescing macrophages could account for the accumulation of the proteins and calcium at the periphery of lipid pools. However, the demonstration that cholesterol may interact with calcium to enhance hydroxyapatite crystal formation and that matrix vesicles, precursors to calcification, are present in atherosclerotic plaques and emanate mainly from VSMCs (30-33) suggest similar processes to those which occur in bone. It is possible that MGP-containing matrix vesicles initiate hydroxyapatite formation and that the calcium bound to OP in the same vicinity facilitates crystal proliferation after release of the hydroxyapatite from the vesicles.

Further indirect evidence for the role of MGP and OP in regulating calcification comes from the observation that both are transcriptionally regulated by vitamin $D_{3}$, the major regulator of calcium in the body. The 1,25-dihydroxyvitamin $\mathrm{D}_{3}$-receptor complex can bind directly to the promoter regions of both genes to activate their transcription $(34,35)$. Vitamin $D_{3}$ can cause rapid calcification of the vasculature in animals, especially if combined with a high cholesterol diet (36).

A number of other bone-associated proteins have been described in vascular lesions including type I collagen, an extracellular matrix protein, SPARC (secreted protein, acidic and rich in cysteine), a glycoprotein capable of binding calcium in the presence of collagen, and bone morphogenetic protein-2a, a protein capable of inducing ectopic bone formation (37-40). The phenomenon of bone metaplasia is well recognized, particularly within the arterial media of vessels from diabetic patients (41). Therefore, further studies are needed to elucidate the relationship between calcification, ossification and expression of these various bone-associated genes in different models of vascular calcification.

\section{Acknowledgments}

We would like to acknowledge the cooperation of the transplant team at Papworth Hospital and the technical assistance of Mrs. J. Horsley. We would also like to thank Dr. R. F. Loeser (The Bowman Gray School of Medicine, Wake University, North Carolina) for the gift of the MGP antibody.

This work was supported by BHF group and project grants to J. C. Metcalfe and P. L. Weissberg.

\section{References}

1. Richardson, P. D., M. J. Davies, and G. V. R. Born. 1989. Influence of plaque configuration and stress distribution on fissuring of coronary atherosclerotic plaques. Lancet. ii:141-144.

2. Loecker, T. H., R. S. Schwartz, C. W. Cotta, and J. R. Hickman. 1992. Fluoroscopic coronary artery calcification and associated coronary disease in asymptomatic young men. JACC (J. Am. Coll. Cardiol.). 19:1167-1172.

3. Mosse, P. R. L., G. R. Campbell, Z. L. Wang, and J. H. Campbell. 1985. Smooth muscle phenotypic expression in human carotid arteries. I. Comparison of cells from diffuse intimal thickenings adjacent to atheromatous plaques with those of the media. Lab. Invest. 53:556-562.

4. Shanahan, C. M., P. L. Weissberg, and J. C. Metcalfe. 1993. Isolation of gene markers of differentiated and proliferating vascular smooth muscle cells. Circ. Res. 73:193-204.

5. Takahashi, K, and B. Nadal-Ginard. 1991. Molecular cloning and sequence analysis of smooth muscle calponin. J. Biol. Chem. 266:13284-13288.

6. Pearlstone, J. R., M. Weber, J. P. Lees-Miller, M. R. Carpenter, and L. B. Smillie. 1987. Amino acid sequence of chicken gizzard smooth muscle SM22 $\alpha$.J. Biol. Chem. 262:5985-5991.

7. Price, P. A., J. D. Fraser, and G. Metz-Virca. 1987. Molecular cloning of matrix Gla protein. Implications for substrate recognition by the vitamin K-dependent $\gamma$-carboxylase. Proc. Natl. Acad. Sci. USA. 84:8335-8339.

8. Oldberg, A., A. Franzen, and D. Heinegard. 1986. Cloning and sequence analysis of rat bone sialoprotein (osteopontin) cDNA reveals an Arg-Gly-Asp cell-binding sequence. Proc. Natl. Acad. Sci. USA. 83:8819-8823.

9. Boehm, T., R. Gonzalez-Sarmiento, M. Kennedy, and T. H. Rabbitts. 1991. A simple technique for generating probes for RNA in situ hybridization: an adjunct to genome mapping exemplified by the $R A G-1 / R A G-2$ gene cluster. Proc Natl. Acad. Sci. USA. 88:3927-3931.

10. Hsu, S. M., L. Raine, and H. Fanger. 1981. The use of avidin-biotin-peroxidase complex $(\mathrm{ABC})$ in immunoperoxidase techniques: a comparison between $\mathrm{ABC}$ and unlabelled antibody (PAP) procedures. J. Histochem. Cytochem. 29:577.

11. Romani, P., N. Bradley, and L. Fletcher. 1990. QBend 10 a new monoclonal antibody to endothelium: assessment of its diagnostic utility in paraffin sections. Histopathology. 17:237-42.

12. Loeser, R., C. S. Carlson, H. Tulli, W. G. Jerome, L. Miller, and R. Wallin. 1992. Articular-cartilage matrix $\gamma$-carboxyglutamic acid-containing protein. Characterization and immunolocatization. Biochem. J. 282:1-6.

13. Giachelli, C. M., N. Bae, M. Almeida, D. T. Denhardt, C. E. Alpers, and S. M. Schwartz. 1993. Osteopontin is elevated during neointima formation in rat arteries and is a novel component of human atherosclerotic plaques. J. Clin. Invest. 92:1686-1696.

14. Giachelli, C., N. Bae, D. Lombardi, M. Majesky, and S. Schwartz. 1991. Molecular cloning and characterization of 2B7, a rat mRNA which distinguishes smooth muscle cell phenotypes in vitro and is identical to osteopontin (secreted phosphoprotein I, 2aR). Biochem. Biophys. Res. Commun. 177:867-873.

15. Gordon, D., M. A. Reidy, E. P. Benditt, and S. M. Schwartz. 1990. Cell proliferation in human coronary arteries. Proc. Natl. Acad. Sci. USA. 87:46004604.

16. O’Brien, E. R., M. R. Garvin, D. K. Stewart, T. Hinohara, J. B. Simpson, S. M. Schwartz, and C. M. Giachelli. 1993. Osteopontin mRNA and protein are overexpressed in human coronary atherectomy specimens: Clues to lesion calcifcation. Circulation. 88:I-619. 
17. Fraser, J. D., and P. A. Price. 1988. Lung, heart and kidney express high levels of mRNA for the vitamin K-dependent matrix Gla protein. J. Biol. Chem. 263:11033-11036.

18. Loeser, R. F., and R. Wallin. 1992. Cell adhesion to matrix Gla protein and its inhibition by an Arg-Gly-Asp-containing peptide. J. Biol. Chem. 267:9459-9462.

19. Miyazaki, Y., M. Setoguchi, S. Yoshida, Y. Higuchi, S. Akizuki, and S. Yamamoto. 1990. The mouse osteopontin gene. Expression in monocytic lineages and complete nucleotide sequence. J. Biol. Chem. 265:14432-14438.

20. Shiraga, H., W. Min, W. J. VanDusen, M. D. Clayman, D. Miner, C. H. Terrell, J. R. Sherbotie, J. W. Foreman, C. Przysiecki, E. G. Neilson, and J. R. Hoyer. 1992. Inhibition of calcium oxalate crystal growth in vitro by uropontin: Another member of the aspartic acid-rich protein superfamily. Proc. Natl. Acad. Sci. USA. 89:426-430.

21. Kohri, K., Y. Suzuki, K. Yoshida, K. Yamamoto, N. Amasaki. T. Yamate, T. Umekawa, M. Iguchi, H. Sinohara, and T. Kurita. 1992. Molecular cloning and sequencing of cDNA encoding urinary stone protein, which is identical to osteopontin. Biochem. Biophys. Res. Comm. 184:859-864.

22. Young, M. F., J. M. Kerr, J. D. Termine, U. M. Wewer, M. G. Wang, O. W. McBride, and L. M. Fisher. 1990. cDNA cloning, mRNA distribution and heterogeneity, chromosomal location, and RFLP analysis of human osteopontin (OPN). Genomics. 7:491-502.

23. Singh, K., M. W. DeVogue, and B. B. Mukherjee. 1990. Physiological properties and differential glycosylation of phosphorylated and nonphosphorylated forms of osteopontin secreted by normal rat kidney cells. J. Biol. Chem. 265:18696-18701.

24. Anderson, H. C. 1983. Calcific diseases: a concept. Arch. Pathol. Lab. Med. 107:341-348.

25. Barone, L. M., T. A. Owen, M. S. Tassinari, R. Bortell, G. S. Stein, and J. B. Lian. 1991. Developmental expression and hormonal regulation of the rat matrix GLA protein (MGP) gene in chondrogenesis and osteogenesis. J. Cell. Biochem. 46:351-365.

26. Hale, J. E., M. K. Williamson, and P. A. Price. 1991. Carboxyl-terminal proteolytic processing of matrix Gla protein. J. Biol. Chem. 266:21145-21149.

27. Lian, J. B., E. L. Prien, M. J. Glimcher, and P. M. Gallop. 1977. The presence of protein-bound $\boldsymbol{\gamma}$-carboxyglutamic acid in calcium-containing renal calculi. J. Clin. Invest. 59:1151-1157.

28. Levy, R. J., J. B. Lian, and P. Gallop. 1979. Atherocalcin, a $\gamma$-carboxyglu- tamic acid containing protein from atherosclerotic plaque. Biochem. Biophys. Res. Commun. 91:41-49.

29. Hunter, G. K., and H. A. Goldberg. 1993. Nucleation of hydroxyapatite by bone sialoprotein. Proc. Natl. Acad. Sci. USA. 90:8562-8565.

30. Hirsch, D., W. J. Landis, R. Azoury, and S. Sarig. 1992. Liposome interactions with hydroxyapatite crystals: A possible mechanism in the calcification of atherosclerotic plaques. Calcif. Tissue Int. 50:261-65.

31. Hirsch, D., R. Azoury, S. Sarig, and H. S. Kruth. 1993. Colocalization of cholesterol and hydroxyapatite in human atherosclerotic lesions. Calcif. Tissue Int. 52:94-98.

32. Kook, K. M. 1976. Calcification of matrix vesicles in human aortic valve and aortic media. Fed. Proc. 35:156-62.

33. Tanimura, A., D. H. McGregor, and H. C. Anderson. 1983. Matrix vesicles in atherosclerotic calcification. Proc. Soc. Exp. Biol. Med. 172:173-177.

34. Cancela, L., C. L. Hsieh. U. Francke, and P. Price. 1990. Molecular structure, chromosome assignment and promoter organization of the human matrix Gla protein gene. J. Biol. Chem. 265:15040-15048.

35. Yoon, K., R. Buenaga, and G. A. Rodan. 1987. Tissue specificity and developmental expression of rat osteopontin. Biochem. Biophys. Res. Commun. 148:1129-1136

36. Toda, T., D. E. Leszczynski, and F. A. Kummerow. 1983. The role of 25-hydroxy-vitamin $\mathrm{D}_{3}$ in the induction of atherosclerosis in swine and rabbit by hypervitaminosis D. Acta. Pathol. Jpn. 33:37-44.

37. Ross, R., and J. A. Glomset. 1976. The pathogenesis of atherosclerosis. $N$. Engl. J. Med. 295:369-377.

38. Raines, E. W., T. F. Lane, M. L. Iruela-Arispe, R. Ross, and E. H. Sage. 1992. The extracellular glycoprotein SPARC interacts with platelet-derived growth factor (PDGF)-AB and -BB and inhibits the binding of PDGF to its receptors. Proc. Natl. Acad. Sci. USA. 89:1281-85.

39. Termine, J. D., H. K. Kleinman, S. W. Whitson, K. M. Conn, M. L. McGarvey, and G. R. Martin. 1981. Osteonectin, a bone-specific protein linking mineral to collagen. Cell. 26:99-105.

40. Bostrom, K., K. E. Watson, S. Horn, C. Wortham, I. M. Herman, and L. L. Demer. 1993. Bone morphogenetic protein expression in human atherosclerotic lesions. J. Clin. Invest. 91:1800-1809.

41. Ferrans, V. J., and J. W. Butany. 1983. Ultrastructural pathology of the heart. In Diagnostic Electron Microscopy. B. F. Trump, and R. T. Jones, editors. John Wiley \& Sons, Inc., New York. 319-441. 